\title{
ON THE DEFINITION OF MEREOLOGICAL CLASS
}

\author{
ROBERT E. CLAY
}

Consider mereology axiomatized as in [1]*. Sobocinski has posed the question, "If the usual definition of class, DMI, is replaced by

$$
[A a] \therefore A \varepsilon \mathrm{KI}(a) . \equiv: A \varepsilon A:[B]: a \subset \operatorname{el}(B) . \equiv . A \varepsilon \mathrm{el}(B),
$$

is the resulting system equivalent to the original?". This note gives a negative answer. Theses $A 12$ and $A 13$, together with the two trivial models which follow them, show where the resulting system is weaker than mereology.

Consider the axiom system A consisting of $A 1-A 6 ; D A 1$.

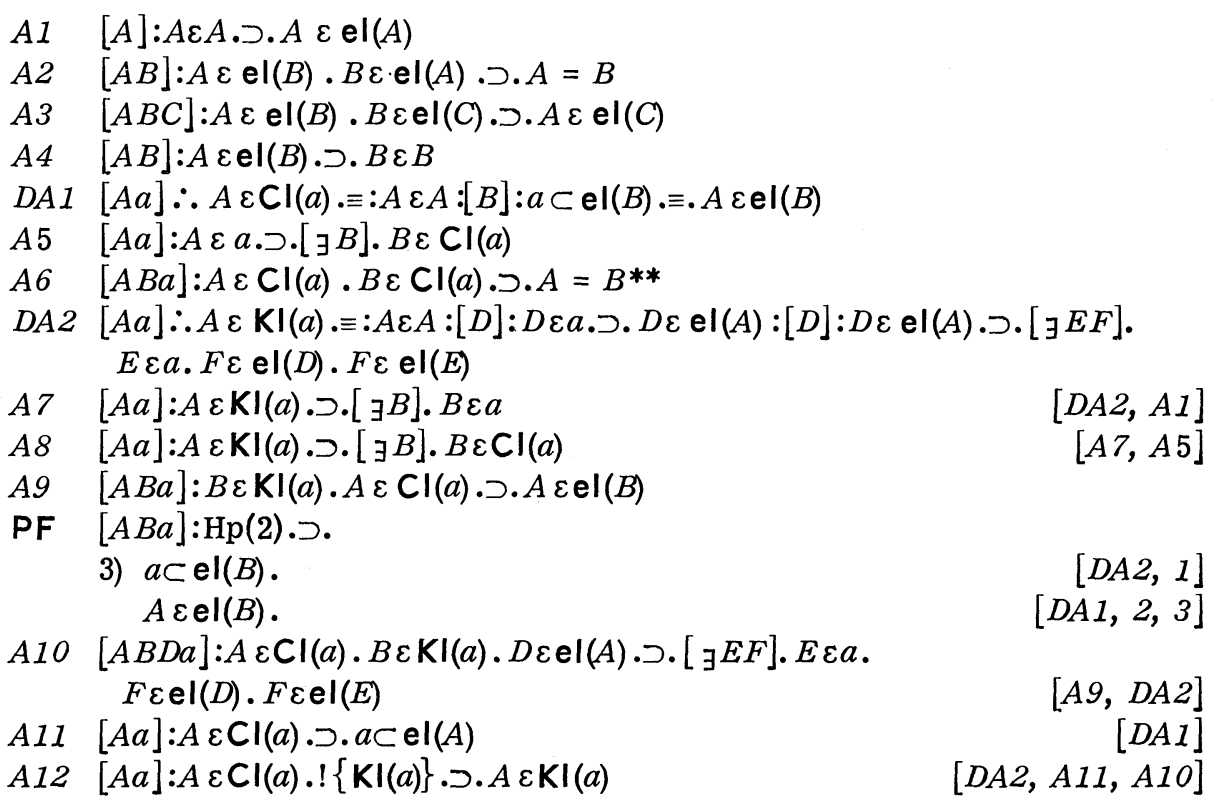

\footnotetext{
*Refer to [1] for the definitions of terms used in this note.

**This system is not independent.
} 
To show that $!\{\mathrm{KI}(a)\}$ may fail, consider the model for A consisting of four names $A, B, C, D$ with the relations, $A \neq B, B \neq C, A \neq C, \operatorname{dscr}\{A \cup B \cup C\}$, $D \varepsilon \mathrm{Cl}(A \cup B \cup C)$. Then $\mathrm{KI}(A \cup B) o \wedge$

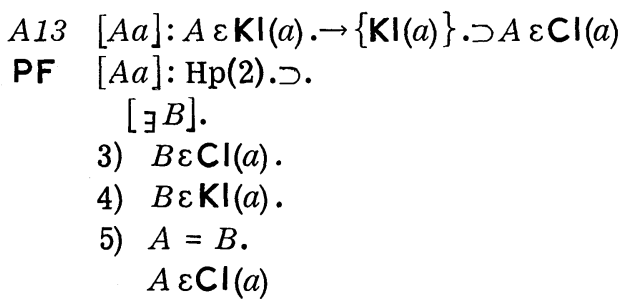

To show that $\rightarrow\{\mathrm{KI}(a)\}$ may fail, consider the model for A consisting of the two names $A, B$ with the relations, $A \neq B, A \varepsilon \mathrm{el}(B)$. Then $A \varepsilon \mathrm{KI}(A)$ and $B \varepsilon \operatorname{KI}(A)$.

\section{RE FERENCE}

[1] R. E. Clay: The relation of weakly discrete to set and equinumerosity in mereology, Notre Dame Journal of Formal Logic, Vol. VI, 1965, pp. 325-340.

University of Notre Dame

Notre Dame, Indiana 